\title{
MR Imaging-Detectable Metabolic Alterations in Attention Deficit/Hyperactivity Disorder: From Preclinical to Clinical Studies
}

\author{
L. Altabella, F. Zoratto, W. Adriani, and R. Canese
}

\begin{abstract}
MR spectroscopy represents one of the most suitable in vivo tool to assess neurochemical dysfunction in several brain disorders, including attention deficit/hyperactivity disorder. This is the most common neuropsychiatric disorder in childhood and adolescence, which persists into adulthood (in approximately $30 \%-50 \%$ of cases). In past years, many studies have applied different MR spectroscopy techniques to investigate the pathogenesis and effect of conventional treatments. In this article, we review the most recent clinical and preclinical MR spectroscopy results on subjects with attention deficit/hyperactivity disorder and animal models, from childhood to adulthood. We found that the most investigated brain regions were the (pre)frontal lobes and striatum, both involved in the frontostriatal circuits and networks that are known to be impaired in this pathology. Neurometabolite alterations were detected in several regions: the NAA, choline, and glutamatergic compounds. The creatine pool was also altered when an absolute quantitative protocol was adopted. In particular, glutamate was increased in children with attention deficit/hyperactivity disorder, and this can apparently be reversed by methylphenidate treatment. The main difficulties in reviewing MR spectroscopy studies were in the nonhomogeneity of the analyzed subjects, the variety of the investigated brain regions, and also the use of different MR spectroscopy techniques. As for possible improvements in future studies, we recommend the use of standardized protocols and the analysis of other brain regions of particular interest for attention deficit hyperactivity disorder, like the hippocampus, limbic structures, thalamus, and cerebellum.
\end{abstract}

ABBREVIATIONS: $\mathrm{ACC}=$ anterior cingulate cortex; $\mathrm{ADHD}=$ attention deficit/hyperactivity disorder; Cho $=$ total choline (phosphorylcholine + glycerolphosphorylcholine); GABA $=\gamma$-aminobutyric acid; PFC $=$ prefrontal cortex; PRESS $=$ point-resolved spectroscopy sequence; $\mathrm{tCr}=$ total creatine $(\mathrm{creatine}+$ phosphorylcreatine)

A ttention deficit/hyperactivity disorder (ADHD) is the most common neurobehavioral disease in children and adolescents. Marked differences in the ADHD prevalence are thought to exist among countries. In fact, the estimated prevalence is $4 \%-7 \%$ in the United States and 1\%-3\% in Europe. ${ }^{1}$ ADHD, which is $2-4$ times more frequent in males than in females, is characterized by traits of inattention, impulsivity, and motor hyperactivity. These can significantly impact many aspects of behavior in children and adolescents and can affect their performance, both at school and at home. ${ }^{2}$

The symptoms of ADHD have a negative impact on the individual throughout childhood, adolescence, and well into adult

Received April 16, 2013; accepted after revision June 14.

From the Department of Cell Biology and Neurosciences (L.A., F.Z., W.A., R.C.), Istituto Superiore di Sanità, Rome, Italy; and Bambino Gesù Children's Hospital IRCCS (F.Z.), Rome, Italy.

This work was funded by ERA-net "NeuroGenMRI" (to W.A.).

Please address correspondence to Rossella Canese, MD, Molecular and Cellular Imaging Division, Department of Cell Biology and Neurosciences, Istituto Superiore di Sanità, viale Regina Elena 299, I-00161 Roma, Italy; e-mail: rossella.canese@iss.it

-- Indicates open access to non-subscribers at www.ajnr.org

http://dx.doi.org/10.3174/ajnr.A3843 life, especially if not managed optimally. In approximately $80 \%$ of children with ADHD, symptoms persist into adolescence and may persist into adulthood in approximately $30 \%-50 \%$ of cases. ADHD adolescents and adults retain from infancy the traits of inattention and impulsivity. Moreover, they start to show a propensity for sensation-seeking and risk-taking and are more likely to develop substance abuse and pathologic dependence from risky activities, including gambling. ADHD is classically considered an executive dysfunction characterized by poor decision-making. ${ }^{2}$ However, it can also be viewed as a motivational dysfunction, arising from altered processing of reward values by the frontostriatal circuits, characterized by attempts to escape or avoid any situation that requires procrastination, such as waiting for the mere elapsing of time, slow gathering of information, active withholding of impulses, and prolonged focusing of attention. ${ }^{1,3}$ From this perspective, ADHD may be a consequence of a psychological inability to give a correct account of and to represent mentally a reward that is not immediately present or accessible. Subjects with ADHD show, as well, diminished concerns for the potential risk of any unforeseen interference and/or negative unexpected outcome. These deficits in cognitive control and/or 
motivation, usually seen in children with ADHD, highlight the importance of the disruption of the dopaminergic and serotonergic systems in this syndrome. ${ }^{4}$ The variability of ADHD subpopulations reported in the medical literature may be partly due to differences in the relative dysfunction between the dopamine and serotonin systems. ${ }^{1,5}$

The diagnosis is based on behavioral observation and semistructured interviews; it does not imply a biologic marker or other objective evidence. Thus, the symptoms of ADHD are difficult to define unequivocally because it is difficult to determine where normal levels of inattention, hyperactivity, and impulsivity end and clinically significant levels (requiring medical intervention) begin. Additionally, the same symptoms can be difficult to differentiate from those indicative of other disorders.

Appropriate management has a significant impact on the symptoms of ADHD. Management options include educational strategies, behavioral treatments, and medical pharmacotherapy. Psychostimulant medications are the treatment of first choice. When nonpharmacologic psychotherapy-based approaches (recommended in those who display mild symptoms) do not succeed in relieving ADHD symptoms and psychostimulant drugs are prescribed, a clear benefit derives from the treatment. Methylphenidate and atomoxetine are the most frequently prescribed drugs for the treatment of ADHD. ${ }^{6,7}$ While such pharmacologic therapy has been shown to improve behavior for the short-term, persistent benefits or adverse effects are largely unknown because of the lack of long-term follow-up studies.

Several brain areas have been identified as abnormal in ADHD. MR imaging offers reliable and noninvasive methods to study in vivo brain morphology and neurochemical compounds.

\section{MR Spectroscopy}

Based on MR imaging, there have been findings of volumetric and functional brain abnormalities in subjects with ADHD reported in the literature. ${ }^{8}$ The volumetric abnormalities involve the frontostriatal network, also with a widespread reduction in the volume of the total cerebrum and cerebellum, in particular for children. More recent reviews considered the dysfunction of the cingulofrontal-parietal cognitive-attention network ${ }^{9}$ as well as a deficit in timing functions ${ }^{10}$ in subjects with ADHD. In this framework, MR spectroscopy can also play an important role in the understanding of the pathogenetic mechanisms that are the basis of this disorder.

Studies of psychiatric diseases are perhaps the most challenging applications of MR spectroscopy because they require stable and reproducible measurements of minute alterations in metabolite concentration. Moreover, MR spectroscopy is a safe, noninvasive in vivo technique for investigating biochemical and metabolic brain features, without the use of radioactive tracers. ${ }^{1} \mathrm{H}-\mathrm{MR}$ spectroscopy can detect and, in particular conditions, quantify several metabolites from a specific brain region. The most relevant MR imaging-detectable neurometabolites are alanine, aspartate, creatine, $\gamma$-aminobutyric acid (GABA), glucose, glutamate, glutamine, glycerol-phosphoryl-choline, guanidoacetate, phosphoryl-choline, myo-Inositol, lactate, $\mathrm{N}$-acetylaspartate, $\mathrm{N}$ acetylaspartylglutamate, phosphor-creatine, scyllo-Inositol, and taurine. At conventional clinical magnetic fields, metabolites that have resonances overlapping or very close are also given as their sum: total choline (Cho; phosphorylcholine + glycerol-phosphorylcholine), Glx (glutamate + glutamine), total creatine (tCr; creatine + phosphorylcreatine).

Different spectroscopic localizing techniques have been developed in the past-from the single-voxel ${ }^{1} \mathrm{H}-\mathrm{MR}$ spectroscopy techniques $^{11}$ to the $2 \mathrm{D}$ or $3 \mathrm{D}$ MR spectroscopy imaging (MR spectroscopy), also called chemical shift imaging. ${ }^{12}$ The former, stimulated echo acquisition mode and point-resolved spectroscopy sequence (PRESS), are the most used MR spectroscopy techniques because they reveal neurometabolites from an a priori chosen localized region. Furthermore, the quantification of metabolite contents is expressed not only as a ratio, usually with respect to $\mathrm{tCr}$, but also as an absolute concentration if a quantitative protocol is applied. ${ }^{13,14}$ On the other hand, chemical shift imaging allows investigating a wider region (a section or a slab) compared with single-voxel techniques as well as comparing different regions of interest within the selected section during postprocessing, but quantification is more critical and usually ratios to tCr are provided. An intrinsic problem of chemical shift imaging is to ensure that selected voxels consist mainly of gray matter because of the partial volume effect. For this reason, corrections for differences in gray and white matter are usually introduced. Peak overlapping prevents revealing some brain metabolites (ie, GABA) that could be of particular interest for specific pathologies. To overcome this problem, GABA spectral editing is a spectroscopic technique that reveals only these metabolites. Spectral editing can be used with spectroscopic techniques such as MEGAPRESS or 2D J-resolved MR spectroscopy and correlated spectroscopy-based methods. ${ }^{15}$

${ }^{31} \mathrm{P}-\mathrm{MR}$ spectroscopy is also applied in the study of brain metabolism: ${ }^{31} \mathrm{P}-\mathrm{MR}$ spectroscopy is less sensitive than ${ }^{1} \mathrm{H} \mathrm{MR}$ spectroscopy, but it provides information about high-energy phosphate metabolism as well as membrane phospholipid synthesis and degradation.

The differences in actually applied methodology and the rare use of a quantitative protocol make it more difficult to review spectroscopy ADHD studies, both in clinical and preclinical settings. Moreover, there are a limited number of ${ }^{31} \mathrm{P}-\mathrm{MR}$ spectroscopy studies.

The aim of this present work was to review the most recent findings in preclinical and clinical studies, conducted on ADHD by using MR spectroscopy.

\section{Why Spectroscopy for ADHD Studies?}

The brain undergoes several metabolic changes during the transition from childhood to adolescence. ${ }^{16,17}$ MR spectroscopy provides a snapshot of these neurochemical alterations and can also be useful in tracking the progress of a neurologic and/or psychiatric disease in longitudinal studies. Several metabolism alterations have indeed been detected in psychiatric disorders such as schizophrenia, ${ }^{18}$ depression, bipolar disorder, ${ }^{19}$ and autism spectrum disorder. ${ }^{20}$ Recent MR spectroscopy studies on ADHD, both at preclinical and clinical levels, have revealed metabolite alterations that can perhaps account for the dopaminergic and/or glutamatergic dysregulation, known to be at the basis of this pathology. $^{21}$ 
The prefrontal cortex (PFC) and the striatum are the most studied regions in subjects with ADHD because these regions belong to the prefrontal striatal thalamo-frontal circuits, well known to be involved in cognition, emotion, and attention processes. $^{9}$

\section{Rodent Models of ADHD}

In behavioral neuroscience, animal models enable the investigation of brain-behavior relations under controlled conditions (eg, standardized housing and testing), with the aim of gaining insight into the neurobiology and pathophysiology of normal and abnormal human behavior and its underlying neuronal and neuroendocrinologic processes. ${ }^{22}$ By exploiting genetic and environmental manipulations, pharmacologic treatments, and so forth, the use of animal models is essential for obtaining information that cannot be gained in other ways. Indeed, in vitro approaches cannot model the interactions in complex systems, such as the brainbehavior relations in live mammals. Thanks to animal models of behavioral dysfunctions, research can lead to both disease prevention and improvements in diagnosis and treatment.

In the development of an animal model of behavioral dysfunction, the concept of validity is of primary importance. ${ }^{22}$ Regarding ADHD, the phenotypic resemblance to human disease (ie, face validity) has been the primary aim for developing animal models of ADHD since the first proposed one, the 6-hydroxydopamine-lesioned rat. ${ }^{23}$ However, the notion that patients with $\mathrm{ADHD}$ can be classified into a predominantly inattentive type, predominantly hyperactive-impulsive type, and combined type, together with preclinical results indicating that inattention, impulsivity, and hyperactivity may not share a common neurobiological substrate, ${ }^{5}$ suggests that an animal model of ADHD does not need to exhibit all the behavioral symptoms of ADHD to possess an adequate face validity.

An animal model that possesses face validity for ADHD may meet other criteria that render it suitable for the study of ADHD. The increase in extracellular dopamine induced by drugs used to treat $\mathrm{ADHD}$ (eg, methylphenidate and atomoxetine) normally produces an increase in motor activity but ameliorates symptoms in patients with ADHD. An animal model of ADHD that shows a similar reduction in symptom expression following drug administration achieves predictive validity.

On the basis of their ability to meet $\geq 1$ of these criteria, $>10$ animal models of ADHD have been developed since the 6-hydroxydopamine-lesioned rat. ${ }^{23}$ The 4 most extensively studied animal models of ADHD are the neonatal 6-hydroxydopaminelesioned rat, the coloboma mutant mouse, the dopamine transporter-knockout/down mouse, and the spontaneously hypertensive rat. The behavioral and neurochemical features of each of these models, with a focus on the features that they share, have been recently reviewed by Fan et al. ${ }^{24}$

The Lenti-dopamine transporter is an innovative and promising animal model, developed through brain inoculation of selfinactivating, regulatable lentiviruses, targeting the dopamine transporter gene and protein expression. ${ }^{25,26}$ Specifically, these animals could serve as a model for symptoms observed in cases of comorbidity between ADHD and pathologic gambling. The Lenti-dopamine transporter model was indeed analyzed in vivo by ${ }^{1} \mathrm{H}$-MR spectroscopy. ${ }^{26}$ Changes among groups were detected mainly in bioenergetic metabolites ( $\mathrm{tCr}$ ), indicating a functional upregulation of the dorsal striatum due to enhancement of the dopamine transporter and, conversely, a downregulation of the ventral striatum (ie, the nucleus accumbens) due to silencing of dopamine transporter. As a result, the rat group, termed "dopamine transporter+silencers," receiving combined dopamine transporter overexpression and silencing within the nucleus accumbens, displayed opposite rearrangement of function within the dorsal-versus-ventral striatum. These 2 regions do respectively subserve an increased habit-based behavior and decreased feedback regulation by evaluation of actual reward. ${ }^{27}$ This profile leads to an impulsive and risk-prone phenotype, whereby the "risk" is represented by a low-feeding payoff. An altered accumbal dopamine transporter function, resulting in a modified dopaminergic tone, may subserve a sensation-seeker phenotype and the vulnerability to impulse-control disorders. ${ }^{25,26}$

Compared with dopamine transporter-knockout and dopamine transporter-knockdown rodents, the principal advantages of Lenti-dopamine transporter rats are localization and inducibility. Further studies on these rats will explore directly the effects of dopamine transporter-targeting drugs currently used in clinics, such as methylphenidate.

From the opposite perspective, an interesting model of reduced impulsivity and decreased risk proneness, which is one characteristic of $\mathrm{ADHD}$, was obtained by exposure of rats to methylphenidate during adolescence. ${ }^{28,29}$ With the aim of characterizing the metabolic forebrain changes induced in adult rats as a consequence of adolescent methylphenidate, a quantitative ${ }^{1} \mathrm{H}-\mathrm{MR}$ spectroscopy analysis was performed in vivo. Most interesting, long-term changes implicated tCr and taurine, metabolites respectively involved in bioenergetics and synaptic efficiency. These were both upregulated in the dorsal striatum and conversely downregulated in the nucleus accumbens of methylphenidate-exposed rats. Unaltered $\mathrm{tCr}$ and an increased phosphorlcreatine/tCr ratio were detected in the $\mathrm{PFC},{ }^{28}$ suggesting enhanced bioenergetics in this area, enabling a better top-down control over subcortical regions. In other words, the capability of the PFC to tune down instinctive drives and tune up elaboration of strategies (ie, self-control) may explain the profile of reduced impulsivity found in the pretreated animals. These findings highlight the role of these MR spectroscopy-detectable metabolites as a marker of functional status within forebrain areas, all of which are well known to subserve the regulation of impulsivity in rats as well as humans.

\section{Clinical MR Spectroscopy Findings: Patients with ADHD}

There are not many clinical MR spectroscopy studies on ADHD in the literature. All the results and methodology we presently reviewed are summarized in the Table.

A recent meta-analysis reviewed all MR spectroscopy studies of ADHD up to 2007. ${ }^{30}$ The authors found, on MEDLINE (PubMed), 16 articles about MR spectroscopy in the prefrontal cortex, striatum, and frontal lobe in ADHD published up to the end of September 2007. Several of these studies revealed metabolic alteration for Cho, NAA, and Glx related to tCr signal in patients with ADHD. The meta-analysis was performed consid- 


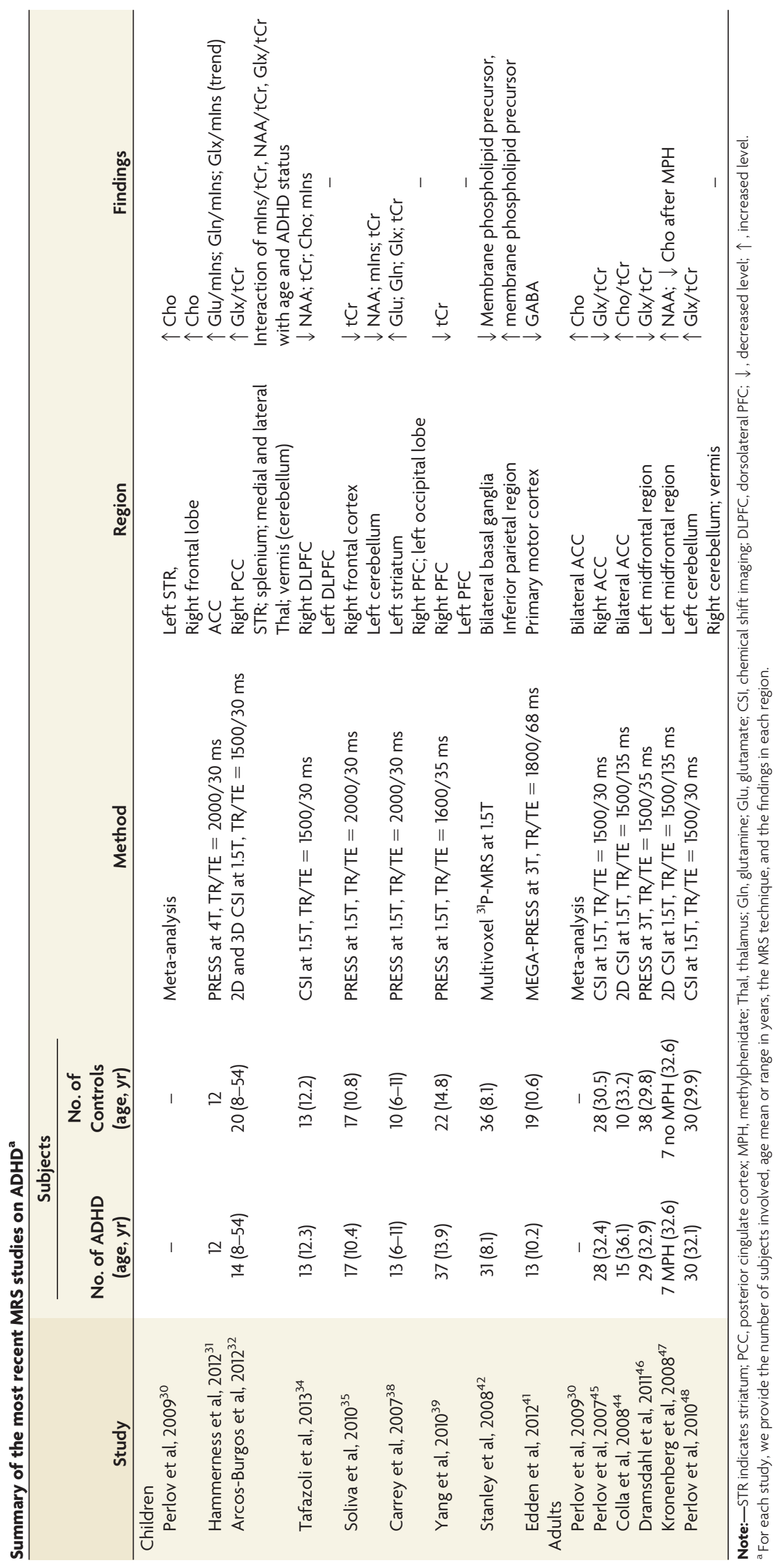


ering metabolites and brain regions separately and led to the finding of an increase in the choline compounds within the left striatum and right frontal lobe for children diagnosed with ADHD.

One recent pilot study ${ }^{31}$ has considered the effect of methylphenidate treatment on metabolite levels with respect to myoInositol in the anterior cingulate cortex (ACC). Ten subjects with ADHD underwent MR spectroscopy before and after a 6- to 8 -week methylphenidate oral treatment at doses of $1.5 \mathrm{mg} / \mathrm{kg} /$ day. A control group of 12 healthy subjects was also analyzed. The main findings were a trend toward increased glutamate/mIns, glutamine/mIns, and $\mathrm{Glx} / \mathrm{mIns}$ levels in subjects with ADHD compared with healthy controls. Moreover, these glutamatergic abnormalities were counteracted by the drug treatment.

Arcos-Burgos et $\mathrm{al}^{32}$ investigated, by using multivoxel 2D and $3 \mathrm{D}$ chemical shift imaging, a variety of brain regions: the striatum, cingulate gyrus, splenium of the corpus callosum, medial and lateral thalamus, and cerebellar vermis. They chose these regions on the basis of a previous study ${ }^{33}$ whereby morphologic abnormalities were reported in subjects with ADHD. The study involved 34 subjects with ADHD (21 females, 8 males) and 20 controls with a wide age range from 8 to 54 years. A significantly higher $\mathrm{Glx} / \mathrm{tCr}$ in the right posterior cingulate cortex of subjects with ADHD was detected. More differences in metabolite ratios were found when considering interactions between metabolite, age, and ADHD status: $\mathrm{mIns} / \mathrm{Cr}$ and $\mathrm{Glx} / \mathrm{tCr}$ in the left posterior cingulate; $\mathrm{NAA} / \mathrm{tCr}$ in the splenium, right posterior cingulate, and left posterior cingulate; and, finally, mIns/tCr and NAA/tCr in the right striatum. These findings suggest that, in ADHD, a different metabolic pattern is followed, with respect to controls, during ontogeny and progression of age.

In 2013, Tafazoli et $\mathrm{al}^{34}$ investigated the bilateral middle frontal gyrus (or dorsolateral prefrontal cortex) in subjects with ADHD ( 8 boys, 5 girls; mean age, 12.3 years) compared with healthy subjects ( 8 boys, 5 girls; mean age, 12.2 years) by short-TE chemical shift imaging by using a quantitative protocol. In addition to short TE and water as internal references, the authors used a self-made software to correct for CSF. They found a significant reduction in NAA, $\mathrm{tCr}$, Cho, and mIns in the right middle frontal gyrus of the ADHD group. This lateralization may reflect a rightsided asymmetry in ADHD. The authors underlined some limitations of this study: the estimated percentage of white (roughly $40 \%$ ) and gray (roughly 50\%) matter within the voxel that can affect quantification and the small and heterogeneous sample. Furthermore, 3 subjects with ADHD were stimulant-treated at the time of the study, though they were medication-free on the day of the MR imaging. This is not a real limitation because a previous study published in $2010^{35}$ found no significant correlations between dose or time length of medication and neurometabolite levels in the frontal gyrus. This research group, by adopting a quantitative protocol, investigated the right frontal cortex and the left cerebellar hemisphere of subjects with ADHD (15 boys, 2 girls; mean age, 10.41 years; mean methylphenidate dose, $30.29 \mathrm{mg} / \mathrm{kg}$; medication time, 18.21 months) and healthy controls ( 15 boys, 2 girls; mean age, 10.76 years). They found a significant reduction of mIns, NAA, and $\mathrm{tCr}$ in the left cerebellum and a reduction of $\mathrm{tCr}$ in the right prefrontal cortex. The reduction in $\mathrm{tCr}$ in both regions had a weaker significance level, and the authors themselves suggested caution in the interpretation of these findings.

\section{Quantitative Protocol}

Beyond the study of Soliva et $\mathrm{al}^{35}$ described above, only a few studies adopted a quantitative protocol to investigate ADHD neurometabolic alterations. The protocol included short TE, water signal as an internal reference, and an LCModel (Stephen Provencher, Oakville, Ontario, Canada) fitting program. The LCModel method analyzes in vivo spectra as a linear combination of model in vitro spectra from individual metabolite solutions, ${ }^{36,37}$ and it is the most widely used software for metabolic spectral quantification. Differences in tissue composition within the voxel can affect the quantification; therefore, it is important to carefully estimate the percentage of gray matter, white matter, and CSF content in the localized region, especially in human studies.

In 2007, Carrey et $\mathrm{al}^{38}$ investigated 13 male subjects with ADHD and 10 healthy male subjects between 6 and 11 years of age by applying short-TE ${ }^{1} \mathrm{H}-\mathrm{MR}$ spectroscopy in the right PFC, left striatum, and left occipital lobe. They found an increase in glutamate, Glx, and $\mathrm{tCr}$ in subjects with ADHD only in the left striatum. Furthermore, the authors investigated subjects with ADHD after 8 weeks of methylphenidate administration. The pharmacologic treatment significantly reduced only the $\mathrm{tCr}$ concentration in subjects with $\mathrm{ADHD}$, but there was a trend toward reduction in striatal glutamate and Glx. These findings are in line with the study of Hammerness et $\mathrm{al}^{31}{ }^{31}$ in which $\mathrm{Glx} / \mathrm{mIns}$ was partially reduced in ACC as an effect of methylphenidate treatment.

A more recent study that includes a quantitative protocol was carried out by Yang et $\mathrm{l}^{39}$ on 37 adolescents with ADHD ( 13 boys, 2 girls; mean age, 13.88 years) and 22 control subjects ( 14 boys, 8 girls; mean age, 14.85 years). The bilateral prefrontal areas of adolescent brains were studied by using short-TE ${ }^{1} \mathrm{H}$-MR spectroscopy. The authors found a reduction in the $\mathrm{tCr}$ level in the right PFC. No sex or age effects were found, probably because the age range of the sample was not enough wide. Here, the authors compared the absolute metabolic levels and their ratio with $\mathrm{tCr}$. With the latter quantification method, NAA/tCr in the right PFC showed a significant increase within the ADHD group, but this finding clearly depends on the reduction of $\mathrm{tCr}$ rather than on increased NAA.

Another study involved the use of methods for absolute metabolite quantification in male adults. ${ }^{40}$ Here, the left dorsolateral PFC and left striatum of 5 unmedicated subject with ADHD (mean age, 27.2 years) and control subjects (mean age, 27.0 years) were analyzed. Most interesting, the authors found a lower concentration of NAA for the ADHD group within the left dorsolateral PFC. No metabolic differences were detected for the striatum.

The alteration in $\mathrm{tCr}$ concentration found in this pathology, as reported by Yang and collegues, ${ }^{39}$ highlights the notion that the use of $\mathrm{tCr}$ as a stable reference for the metabolite relative quantification is not correct.

More recently, the GABA-editing technique allowed investigation of the dysregulation of this important neurotransmitter in ADHD. Edden et $\mathrm{al}^{41}$ studied the primary motor cortex of 13 children (11 boys, 2 girls; mean age, 10.2 years) diagnosed with ADHD compared with 19 control subjects ( 12 boys, 7 girls; mean 
age, 10.6 years). Using MEGA-PRESS for GABA editing, they reported a significant reduction in the levels of this neurotransmitter for the subjects with ADHD.

\section{${ }^{31}$ P-MR Spectroscopy \\ ${ }^{31} \mathrm{P}$-MR spectroscopy is not yet widely used because it necessitates dedicated equipment (coils), a long acquisition time, and addi- tional costs. On the other hand, ${ }^{31} \mathrm{P}-\mathrm{MR}$ spectroscopy provides important information about the metabolism of phosphate com- pounds and the cellular energy metabolism. \\ Only two studies ${ }^{42,43}$ have reported ${ }^{31} \mathrm{P}-\mathrm{MR}$ spectroscopy data in patients with ADHD. In the most recent article, ${ }^{42}$ Stanley et al compared 31 children with ADHD with 36 control subjects by using multivoxel ${ }^{31} \mathrm{P}-\mathrm{MR}$ spectroscopy to find alterations in membrane phospholipid precursors. These included phosphomo- noesters, phosphoethanolamine, and phosphocholine, which are precursors of membrane phospholipids, and the phosphodiesters, glycerophosphoethanolamine and glycerophosphocholine, which are breakdown products of membrane phospholipids. The results showed a significant reduction of the membrane phospholipid pre- cursor in the bilateral basal ganglia and, conversely, an increase in the right inferior parietal lobe.}

\section{Adults}

There are still few MR spectroscopy studies on adults with ADHD. In the meta-analysis of $2009,{ }^{30}$ only 3 studies were considered. ${ }^{40,44,45}$ The work of Hesslinger et $\mathrm{al}^{40}$ has already been discussed in the quantification section, and it is the only study that involved the left dorsolateral PFC and left striatum. The other 2 studies involved the bilateral ACC. Perlov et $\mathrm{al}^{45}$ found Glx/tCr to be decreased in the right ACC of 28 subjects with ADHD ( 17 men, 11 women; mean age, 32.4 years) compared with 28 controls (15 men, 13 women; mean age, 30.5 years). This last finding is in line with the study of Colla et al, ${ }^{44}$ in which 15 patients with ADHD ( 8 men, 7 women) were compared with 10 healthy controls ( 4 men, 6 women). Most interesting, these authors found an increase in choline compounds with respect to tCr not only in the right ACC but also in the left one. No alterations in glutamatergic metabolites were found, mainly because these authors used a long TE (135 ms).

Also, a more recent study ${ }^{46}$ considered the bilateral midfrontal region, including the ACC, in ADHD pathology. This study compared the metabolite level ratio with $\mathrm{tCr}$ in 29 adults with ADHD (15 men, 14 women; mean age, 32.9 years) with respect to 38 healthy controls ( 15 men, 23 women; mean age, 29.8 years). The main finding was a reduction in $\mathrm{Glx} / \mathrm{tCr}$ within the left midfrontal region. In the same region, Kronenberg et $\mathrm{al}^{47}$ found, in 7 adults with ADHD, an increase in NAA and a reduction in Cho after a chronic (5-6 weeks) methylphenidate treatment but no change at all in glutamate compounds.

In 2010, Perlov et $\mathrm{al}^{48}$ investigated the whole cerebellum, covering the vermis and the 2 hemispheres, of 30 patients with ADHD (18 men, 12 women; mean age, 32.1 years) and 30 healthy subjects ( 15 men and 15 women; mean age, 29.9 years) by using multivoxel MR spectroscopy. The Glx level with respect to $\mathrm{tCr}$ was significantly higher in the left cerebellar hemisphere in the ADHD group.

\section{DISCUSSION}

All the investigated regions in the above-mentioned MR spectroscopy studies have an important role in ADHD pathology.

While the dorsal striatum is classically thought to subserve innate or habit-based behavior, the ventral striatum (nucleus accumbens) is involved in reinforcement processes, subserving incentive motivation. ${ }^{49}$ Specifically, the latter accounts for the affective evaluation of distinctive features in the outcome of one's own actions and for feedback modulation of future choice. ${ }^{50}$ Sensation- and risk-seeking may result from either an over- or underactive nucleus accumbens, which may lead to a specific proneness toward an impulsive phenotype and/or gambling behaviors. ${ }^{51}$

The PFC may have a functional role in controlling (or causing) motor and cognitive impulsivity, being involved in the resolution of conflicting decisions through planning, feedback regulation, and inhibition of behavior. ${ }^{52}$ Recent advances in ADHD research propose that the PFC is important for sustaining and shifting attention in an appropriate manner; screening sources of distractions; and inhibiting inappropriate emotions, impulses, and habits. ${ }^{53}$ In more detail, the medial PFC is involved in the following: 1) monitoring of actions and their outcomes, for guiding decisions; and 2) goal-related planning, problem solving, and prediction of forthcoming events. ${ }^{54}$ The orbital PFC is implicated in stimulus-reward associations, their change with time (ie, reversal learning), and in subjective attribution of reward value for choices involving a delayed reinforcement. ${ }^{52}$ Indeed, by acting together, these 2 subdivisions direct behavior more efficiently than would be observed if subcortical drives, elicited within the dorsal and ventral striata, were left alone with no cortical control. ${ }^{55}$

The ACC and dorsolateral PFC are the main components of the cingulofrontal parietal cognitive-attention network. ${ }^{9}$ With 7 studies in pediatric and 5 in adult subjects with ADHD, they are the most analyzed regions. The importance of the ACC in adults $^{44,46,48}$ is also indicated by a recent meta-analysis ${ }^{56}$ on structural MR imaging findings in children and adults with ADHD. Indeed, that work reveals a decreased gray matter volume in the left ACC, but only in adults. Three studies in pediatric and 1 in adult patients with ADHD involved the striatum and basal ganglia, another node of the attention and cognition network. Recently, the cerebellum became a region of interest, so far with one pediatric study and another in adult subjects with ADHD.

Several metabolic alterations have been found in the reviewed studies: Glx, NAA, Cho, and tCr.

Glutamatergic metabolites (glutamate, glutamine) were found to be altered in ADHD: They increase within the ACC, right posterior cingulate cortex, and left striatum in pediatric ADHD, whereas they decrease within the right ACC, left midfrontal regions, and left cerebellar hemisphere in adult ADHD. ${ }^{31,32,38,45,46,48}$ Glutamate is the principal excitatory neurotransmitter in the brain, and it plays a central role in neurotransmission, especially within the ACC where high glutamate levels have been associated with impulsivity. ${ }^{57} \mathrm{MR}$ spectroscopy findings in $\mathrm{ADHD}$ are in agreement with the hypothesis of Carlsson $^{58}$ of a deep glutamate/dopamine interaction, especially within the prefrontal circuits. A hypofunctionality within the do- 
paminergic system can lead to higher glutamate concentrations in pediatric subjects with $\mathrm{ADHD}$, which can be partially reverted by methylphenidate administration, ${ }^{31,38}$ which increases the dopamine levels. In adults, glutamatergic decrease ${ }^{45,46}$ within the midfrontal region, including the ACC, may contribute to the cognitive deficit.

Following the hypothesis of Todd and Botteron, ${ }^{59}$ according to which ADHD could be considered an energy-deficit syndrome, altered energy metabolism of monoamines can cause the excess in glutamate, which is then not correctly absorbed into astrocytes. In this framework, the alterations found for the $\mathrm{tCr}$ (as detected by ${ }^{1} \mathrm{H}-\mathrm{MR}$ spectroscopy) and for phosphorlcreatine (by ${ }^{31} \mathrm{P}-\mathrm{MR}$ spectroscopy), which are metabolites related to bioenergetics of the human brain, can also be explained. Alterations in the Cr pool, as found in absolute quantitative studies, are also important because this notion implies that this metabolite is not a stable reference. As such, more caution should, therefore, be taken in relative quantitative studies in the choice of the reference. As a recommendation for future works, the use of absolute quantitative protocols is desirable.

GABA reduction ${ }^{41}$ suggests that more neurotransmitter systems than previously thought are involved in ADHD. In particular, GABA concentration correlates with impulsive behavior. ${ }^{60}$

The decrease of NAA in the dorsolateral PFC and cerebellum of pediatric subjects ${ }^{34,35}$ and in the dorsolateral PFC of adults ${ }^{40}$ does not necessarily mean a neuronal cell loss, but it can also be interpreted as a neuronal dysfunction. ${ }^{61}$ This metabolic reduction is reversed in adults by methylphenidate administration. ${ }^{47}$ Here, despite the small size of the sample, a stimulant treatment significantly decreased the ACC Cho level, while it increased NAA in adult methylphenidate-responsive patients with ADHD. The increase in NAA can be due to mitochondrial respiratory chain enzyme activities induced by chronic methylphenidate exposure, especially in the PFC and striatum. ${ }^{62}$

Choline-containing compounds are the main components of cell membranes and products of membrane degradation. Changes in Cho are associated with acute demyelinating disease $e^{63}$ or cellular turnover. ${ }^{64}$ Choline is the precursor of acetyl-choline and influences the neural communication, mediated by transmitters like norepinephrine and dopamine. Alterations in choline compounds are observed in only a few of the above-mentioned works and cannot yet be correctly interpreted in the ADHD context.

\section{CONCLUSIONS}

All the reviewed studies give a deeper insight into the ADHD mechanisms. Neurochemical alterations found in several brain regions are in agreement with the most recent hypotheses regarding the pathogenetic processes at the basis of this pathology.

Methodologic improvements of MR spectroscopy procedures are desirable to evaluate the absolute concentrations rather than ratios. There is the need not only for standardized protocols to compare data acquired in different centers but also for smaller voxel sizes, to have high tissue purity and more spatial resolution. On the other hand, this approach would lead to a longer scanning time, which is problematic for subjects with ADHD. Starting from functional results and neurobiologic knowledge, other regions should be investigated with both ${ }^{1} \mathrm{H}$ and possibly ${ }^{31} \mathrm{P}-\mathrm{MR}$ spectroscopy. These regions include the amygdala and the other limbic structures, which are known to play a key role in symptoms like impulsivity. Also, the hippocampus would need to be investigated as well as the thalamus and cerebellum (the latter being involved in a few studies up to now).

Finally, to improve the MR spectroscopy analyses, a balanced choice of subjects in the clinical samples, as far as age, sex, and psychiatric diagnosis are concerned, would be desirable.

Disclosures: Walter Adriani-RELATED: Grant: Italian Ministry of Health, ${ }^{*}$ Comments: ERA-net "NeuroGenMRI," UNRELATED: Patents (planned, pending or issued): Istituto Superiore di Sanità (50\%), ${ }^{*}$ Geropharm Ltd (50\%), ${ }^{*}$ Comments: O. Granstrem, W. Adriani, G. Laviola, M.C. Porfirio, P. Curatolo-provisional patent "Biomarkers for Validation of ADHD (Attention Deficit and Hyperactivity Disorder) Diagnosis and Monitoring of Therapy Efficacy" US Patent and Trademark Office EFS ID 13464574; Application No. 61681638 (10-AUG-2012). *Money paid to the institution.

\section{REFERENCES}

1. Oades RD. Frontal, temporal and lateralized brain function in children with attention-deficit hyperactivity disorder: a psychophysiological and neuropsychological viewpoint on development. Behav Brain Res 1998;94:83-95

2. Castellanos FX, Sonuga-Barke EJ, Milham MP, et al. Characterizing cognition in ADHD: beyond executive dysfunction. Trends Cogn Sci 2006;10:117-23

3. Sonuga-Barke EJ. Causal models of attention-deficit/hyperactivity disorder: from common simple deficits to multiple developmental pathways. Biol Psychiatry 2005;57:1231-38

4. Casey BJ, Durston S. From behavior to cognition to the brain and back: what have we learned from functional imaging studies of attention deficit hyperactivity disorder? Am J Psychiatry 2006;163: 957-60

5. Sagvolden T, Aase $\mathrm{H}$, Zeiner $\mathrm{P}$, et al. Altered reinforcement mechanisms in attention-deficit/hyperactivity disorder. Behav Brain Res 1998;94:61-71

6. Accardo P, Blondis TA. What's all the fuss about Ritalin? J Pediatr 2001;138:6-9

7. Scheffler RM, Hinshaw SP, Modrek S, et al. The global market for ADHD medications. Health Aff (Millwood) 2007;26:450-57

8. Altabella L, Strolin S, Villani N, et al. Magnetic resonance imaging and spectroscopy in the study in ADHD syndrome: a short review. Biophysics and Bioeng Letters 2011;4:23-34

9. Bush G. Cingulate, frontal, and parietal cortical dysfunction in attention-deficit/hyperactivity disorder. Biol Psychiatry 2011;69: $1160-67$

10. Hart H, Radua J, Mataix-Cols D, et al. Meta-analysis of fMRI studies of timing in attention-deficit hyperactivity disorder (ADHD). Neurosci Biobehav Rev 2012;36:2248-56

11. de Graaf RA. In Vivo NMR Spectroscopy: Principles and Techniques. Chichester, UK: John Wiley; 2008

12. Posse S, Otazo R, Dager SR, et al. MR spectroscopic imaging: principles and recent advances. J Magn Reson Imaging 2013;37:1301-25

13. Canese R, Pisanu ME, Mezzanzanica D, et al. Characterisation of in vivo ovarian cancer models by quantitative $1 \mathrm{H}$ magnetic resonance spectroscopy and diffusion-weighted imaging. NMR Biomed 2012; 25:632-42

14. Scheidegger O, Wingeier K, Stefan D, et al. Optimized quantitative magnetic resonance spectroscopy for clinical routine. Magn Reson Med 2013;70:25-32

15. Puts NA, Edden RA. In vivo magnetic resonance spectroscopy of GABA: a methodological review. Prog Nucl Magn Reson Spectrosc 2012;60:29-41

16. Blüml S, Wisnowski JL, Nelson MD Jr, et al. Metabolic maturation of the human brain from birth through adolescence: insights from in 
vivo magnetic resonance spectroscopy. Cereb Cortex 2013;23: $2944-55$

17. Horská A, Kaufmann WE, Brant LJ, et al. In vivo quantitative proton MRSI study of brain development from childhood to adolescence. $J$ Magn Reson Imaging 2002;15:137-43

18. Vyas NS, Patel NH, Herscovitch P, et al. Recent developments in neurochemical imaging in schizophrenia: an update. Curr Med Chem 2013;20:351-56

19. Kraguljac NV, Reid M, White D, et al. Neurometabolites in schizophrenia and bipolar disorder: a systematic review and meta-analysis. Psychiatry Res 2012;203:111-25

20. Baruth JM, Wall CA, Patterson MC, et al. Proton magnetic resonance spectroscopy as a probe into the pathophysiology of autism spectrum disorders (ASD): a review. Autism Res 2013;6:119-33

21. Biederman J, Faraone SV. Attention-deficit hyperactivity disorder. Lancet 2005;366:237-48

22. van der Staay FJ. Animal models of behavioral dysfunctions: basic concepts and classifications, and an evaluation strategy. Brain Res Rev 2006;52:131-59

23. Shaywitz BA, Yager RD, Klopper JH. Selective brain dopamine depletion in developing rats: an experimental model of minimal brain dysfunction. Science 1976;191:305-08

24. Fan X, Bruno KJ, Hess EJ. Rodent models of ADHD. Curr Top Behav Neurosci 2012;9:273-300

25. Adriani W, Boyer F, Gioiosa L, et al. Increased impulsive behavior and risk proneness following lentivirus-mediated dopamine transporter over-expression in rats' nucleus accumbens. Neuroscience 2009;159:47-58

26. Adriani W, Boyer F, Leo D, et al. Social withdrawal and gamblinglike profile after lentiviral manipulation of DAT expression in the rat accumbens. Int J Neuropsychopharmacol 2010;13:1329-42

27. Adriani W, Zoratto F, Laviola G. Brain processes in discounting: consequences of adolescent methylphenidate exposure. Curr Top Behav Neurosci 2012;9:113-43

28. Adriani W, Canese R, Podo F, et al. 1H MRS-detectable metabolic brain changes and reduced impulsive behavior in adult rats exposed to methylphenidate during adolescence. Neurotoxicol Teratol 2007;29:116-25

29. Adriani W, Leo D, Greco D, et al. Methylphenidate administration to adolescent rats determines plastic changes on reward-related behavior and striatal gene expression. Neuropsychopharmacology 2006; 31:1946-56

30. Perlov E, Philipsen A, Matthies S, et al. Spectroscopic findings in attention-deficit/hyperactivity disorder: review and meta-analysis. World J Biol Psychiatry 2009;10:355-65

31. Hammerness P, Biederman J, Petty C, et al. Brain biochemical effects of methylphenidate treatment using proton magnetic spectroscopy in youth with attention-deficit hyperactivity disorder: a controlled pilot study. CNS Neurosci Ther 2012;18:34-40

32. Arcos-Burgos M, Londono AC, Pineda DA, et al. Analysis of brain metabolism by proton magnetic resonance spectroscopy $(1 \mathrm{H}-$ MRS) in attention-deficit/hyperactivity disorder suggests a generalized differential ontogenic pattern from controls. Atten Defic $\mathrm{Hy}$ peract Disord 2012;4:205-12

33. Krain AL, Castellanos FX. Brain development and ADHD. Clin Psychol Rev 2006;26:433-44

34. Tafazoli S, O'Neill J, Bejjani A, et al. 1H MRSI of middle frontal gyrus in pediatric ADHD. J Psychiatr Res 2013;47:505-12

35. Soliva JC, Moreno A, Fauquet J, et al. Cerebellar neurometabolite abnormalities in pediatric attention/deficit hyperactivity disorder: a proton MR spectroscopic study. Neurosci Lett 2010;470:60-64

36. Provencher SW. Estimation of metabolite concentrations from localized in vivo proton NMR spectra. Magn Reson Med 1993;30: 672-79

37. Provencher SW. Automatic quantitation of localized in vivo $1 \mathrm{H}$ spectra with LCModel. NMR Biomed 2001;14:260-64

38. Carrey NJ, MacMaster FP, Gaudet L, et al. Striatal creatine and glutamate/glutamine in attention-deficit/hyperactivity disorder. J Child Adolesc Psychopharmacol 2007;17:11-17

39. Yang P, Wu MT, Dung SS, et al. Short-TE proton magnetic resonance spectroscopy investigation in adolescents with attentiondeficit hyperactivity disorder. Psychiatry Res 2010;181:199-203

40. Hesslinger B, Thiel T, Tebartz van Elst L, et al. Attention-deficit disorder in adults with or without hyperactivity: where is the difference? A study in humans using short echo (1)H-magnetic resonance spectroscopy. Neurosci Lett 2001;304:117-19

41. Edden RA, Crocetti D, Zhu H, et al. Reduced GABA concentration in attention-deficit/hyperactivity disorder. Arch Gen Psychiatry 2012;69:750-53

42. Stanley JA, Kipp H, Greisenegger E, et al. Evidence of developmental alterations in cortical and subcortical regions of children with attention-deficit/hyperactivity disorder: a multivoxel in vivo phosphorus 31 spectroscopy study. Arch Gen Psychiatry 2008;65:1419-28

43. Stanley JA, Kipp H, Greisenegger E, et al. Regionally specific alterations in membrane phospholipids in children with ADHD: an in vivo 31P spectroscopy study. Psychiatry Res 2006;148:217-21

44. Colla M, Ende G, Alm B, et al. Cognitive MR spectroscopy of anterior cingulate cortex in ADHD: elevated choline signal correlates with slowed hit reaction times. J Psychiatr Res 2008;42:587-95

45. Perlov E, Philipsen A, Hesslinger B, et al. Reduced cingulate glutamate/glutamine-to-creatine ratios in adult patients with attention deficit/hyperactivity disorder: a magnet resonance spectroscopy study. J Psychiatr Res 2007;41:934-41

46. Dramsdahl M, Ersland L, Plessen KJ, et al. Adults with attentiondeficit/hyperactivity disorder: a brain magnetic resonance spectroscopy study. Front Psychiatry 2011;2:65

47. Kronenberg G, Ende G, Alm B, et al. Increased NAA and reduced choline levels in the anterior cingulum following chronic methylphenidate: a spectroscopic test-retest study in adult ADHD. Eur Arch Psychiatry Clin Neurosci 2008;258:446-50

48. Perlov E, Tebarzt van Elst L, Buechert M, et al. H(1)-MR-spectroscopy of cerebellum in adult attention deficit/hyperactivity disorder. J Psychiatr Res 2010;44:938-43

49. Yin HH, Knowlton BJ, Balleine BW. Lesions of dorsolateral striatum preserve outcome expectancy but disrupt habit formation in instrumental learning. Eur J Neurosci 2004;19:181-89

50. Cardinal RN, Winstanley CA, Robbins TW, et al. Limbic corticostriatal systems and delayed reinforcement. Ann N Y Acad Sci 2004; 1021:33-50

51. Bjork JM, Knutson B, Fong GW, et al. Incentive-elicited brain activation in adolescents: similarities and differences from young adults. J Neurosci 2004;24:1793-802

52. Dalley JW, Cardinal RN, Robbins TW. Prefrontal executive and cognitive functions in rodents: neural and neurochemical substrates. Neurosci Biobehav Rev 2004;28:771-84

53. Arnsten AF. Toward a new understanding of attention-deficit hyperactivity disorder pathophysiology: an important role for prefrontal cortex dysfunction. CNS Drugs 2009;23(suppl 1):33-41

54. Seamans JK, Lapish CC, Durstewitz D. Comparing the prefrontal cortex of rats and primates: insights from electrophysiology. $\mathrm{Neu}$ rotox Res 2008;14:249-62

55. Ridderinkhof KR, van den Wildenberg WP, Segalowitz SJ, et al. Neurocognitive mechanisms of cognitive control: the role of prefrontal cortex in action selection, response inhibition, performance monitoring, and reward-based learning. Brain Cogn 2004;56:129-40

56. Frodl T, Skokauskas N. Meta-analysis of structural MRI studies in children and adults with attention deficit hyperactivity disorder indicates treatment effects. Acta Psychiatr Scand 2012;125: $114-26$

57. Hoerst M, Weber-Fahr W, Tunc-Skarka N, et al. Correlation of glutamate levels in the anterior cingulate cortex with self-reported impulsivity in patients with borderline personality disorder and healthy controls. Arch Gen Psychiatry 2010;67:946-54

58. Carlsson ML. On the role of prefrontal cortex glutamate for the 
antithetical phenomenology of obsessive compulsive disorder and attention deficit hyperactivity disorder. Prog Neuropsychopharmacol Biol Psychiatry 2001;25:5-26

59. Todd $\mathrm{RD}$, Botteron $\mathrm{KN}$. Is attention-deficit/hyperactivity disorder an energy deficiency syndrome? Biol Psychiatry 2001;50:151-58

60. Silveri MM, Sneider JT, Crowley DJ, et al. Frontal lobe gammaaminobutyric acid levels during adolescence: associations with impulsivity and response inhibition. Biol Psychiatry 2013;74: 296-304

61. Dautry C, Vaufrey F, Brouillet E, et al. Early N-acetylaspartate deple- tion is a marker of neuronal dysfunction in rats and primates chronically treated with the mitochondrial toxin 3-nitropropionic acid. J Cereb Blood Flow Metab 2000;20:789-99

62. Fagundes AO, Rezin GT, Zanette F, et al. Chronic administration of methylphenidate activates mitochondrial respiratory chain in brain of young rats. Int J Dev Neurosci 2007;25:47-51

63. De Stefano N, Filippi M. MR spectroscopy in multiple sclerosis. J Neuroimaging 2007;17(suppl 1):31S-35S

64. Gujar SK, Maheshwari S, Bjorkman-Burtscher I, et al. Magnetic resonance spectroscopy. J Neuroophthalmol 2005;25:217-26 